\title{
Clinical Implications of Congenital Absence of Circumflex Coronary Artery
}

\author{
Ahmet Karabulut ${ }^{*}$ \\ 'Department of Cardiology, School of Medicine, Acibadem Mehmet Ali Aydınlar University, Istanbul, Turkey \\ *Corresponding Author: Ahmet Karabulut, M.D., Associate Professor, Acibadem Atakent Hospital, Department of \\ Cardiology, Turgut Ozal Bulvarı, No: 16, 34303, Istanbul, Turkey. Tel: +90-5053577477, Fax: +90-212-4044445, \\ Email: drkarabulut@yahoo.com
}

Received August 4, 2017; Accepted August 29, 2017; Online Published September 4, 2017

\begin{abstract}
Introduction: Coronary artery anomalies are rare clinical entities reported in $0.6 \%$ to $5.6 \%$ of diagnostic coronary angiographies. Anomalous origins of coronary arteries from distal segments are rarely reported. Herein, the presented case is a coronary anomaly in which the circumflex artery (CX) originated as a continuation of the posterolateral branch of the right coronary artery (RCA) with separate left anterior descending (LAD) artery origination from the left sinus of Valsalva. The clinical presentation of such a rare anomaly is discussed, and the current literature regarding the congenital absence of $\mathrm{CX}$ is reviewed.

Case Presentation: A 66-year-old male presented with angina pectoris. Coronary angiography revealed critical stenosis in the mid segment of the LAD artery and an anomalous origin of CX artery from the distal RCA. The CX was coursing as a continuation of the posterolateral branch of the RCA. Coronary angioplasty and stent deployment was performed for the LAD lesion, and the patient was discharged with medical therapy. The patient did not report recurrent anginal symptoms under medical therapy.

Conclusion: The congenital absence of the circumflex coronary artery is a rare coronary anomaly. The clinical presentation may vary, though most cases are asymptomatic during diagnosis. Such cases require close clinical follow-up since they entertain a future risk for being symptomatic, especially in the setting of atherosclerotic disease.

Keywords: Coronary Vessel Anomalies, Incidence, Symptom Assessment, Prognosis
\end{abstract}

\section{Introduction}

Anomalous origin of the circumflex artery (CX) from the proximal portion of the right coronary artery (RCA) or right sinus of Valsalva has been reported as the second most common coronary anomaly. ${ }^{1}$ On the contrary, congenital absence of the CX is an extremely rare clinical occasion. In such cases, the RCA is almost always well-developed and can be described as the superdominant RCA. The CX usually arises from the distal RCA.,3 Based on the size of the CX and perfusion of the circumflex territory, clinical symptoms may vary from an asymptomatic condition to an acute coronary syndrome. Herein, a case in which CX originated as a continuation of the posterolateral branch of the RCA with a separate left anterior descending (LAD) artery origination from the left sinus of Valsalva. The clinical implication of this anomaly is discussed.

\section{Case Presentation}

A 66-year-old male presented with a Canadian Cardiovascular Society (CCS) class 3 angina pectoris. The patient was a heavy smoker. His physical examination, electrocardiogram (ECG), and echocardiography were unremarkable. Coronary angiography revealed critical stenosis in the mid section of the LAD artery (Figure 1). An anomalous origin of the CX artery from the distal RCA was also observed. The CX was coursing as a continuation of the posterolateral branch of the RCA, and its body and branches extended to the border of the proximal LAD (Figure 2). Coronary angioplasty and stent deployment was performed for the LAD lesion, and the patient was discharged with medical therapy. He did not report recurrent anginal symptoms under medical therapy.

\section{Discussion}

Coronary artery anomalies are rare clinical entities reported in only $0.6 \%$ to $5.6 \%$ of diagnostic coronary angiographies. ${ }^{1}$ Most are asymptomatic and incidental findings which do not require additional treatment. The origination of the CX from the proximal RCA and right sinus of Valsalva is the second most common coronary

Copyright (C) 2017 The Author(s). This is an open-access article distributed under the terms of the Creative Commons Attribution License (http:// creativecommons.org/licenses/by/4.0), which permits unrestricted use, distribution, and reproduction in any medium, provided the original work is properly cited. 


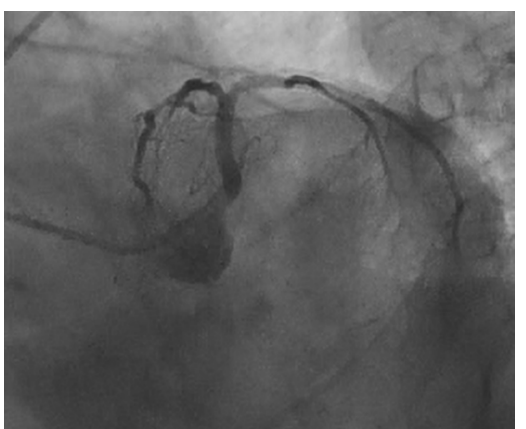

Figure 1. Spider View Showing Separately Originating Left Anterior Descending Artery With Discrete Diagonal Branch and Absence of Circumflex Artery.

anomaly after the separate origins of LAD and CX $(0.37 \%$ vs $0.41 \%){ }^{1,2}$ The anomalous origin of coronary arteries from distal segments has been reported only rarely. In the majority of reported cases, the RCA originated from the distal $\mathrm{CX},{ }^{4,5}$ whereas CX originating from the distal RCA has been reported in only a few cases associated with a single coronary anomaly. ${ }^{2,3}$ The congenital absence of the left CX is also rarely reported. In such cases, the posterolateral branch of the RCA is usually well-developed, and it courses to the circumflex territory. Such a large vessel is defined as a superdominant RCA and has an incidence rate of $0.003 \%$ to $0.067 \%{ }^{6}$

A coronary artery anomaly is defined as a variant of the normal coronary anatomy. The majority of cases are usually diagnosed incidentally, and nearly $80 \%$ of all cases are asymptomatic. Due to the benign prognosis, such cases do not require additional treatment. ${ }^{5}$ However, an acute coronary syndrome related to a coronary anomaly has a more serious progression because of the extensive territory to which blood is supplied. ${ }^{7,8}$ Moreover, percutaneous coronary intervention for a coronary anomaly is a challenging procedure because of the complex anatomy and higher associated clinical risk which necessitates the expertise of an experienced interventional cardiologist. ${ }^{9}$

Several case presentations have demonstrated different clinical pictures in the congenital absence of the CX. Physiological or mental stress or exercise may trigger stable angina pectoris, especially in a fairly coursing CX. In the setting of atherosclerotic disease, anginal attacks are observed more frequently. An especially slow coronary flow could lead to delayed perfusion of the circumflex territory, which may be more evident after a heavy meal or smoking. Moreover, acute angulation of the vessel may lead to similar cilinical pictures of myocardial bridging. ${ }^{10}$ In the present case, the CX was well-developed; thus, symptomatology was linked to a LAD lesion. After successful intervention, the patient did not report recurrent angina. However, the patient was under anti-ischemic medication which may have suppressed the symptoms of the CX anomaly. Myocardial perfusion scintigraphy may be useful in detecting ischemic regions of the CX territory, and this may enable doctors to prescribe more accurate

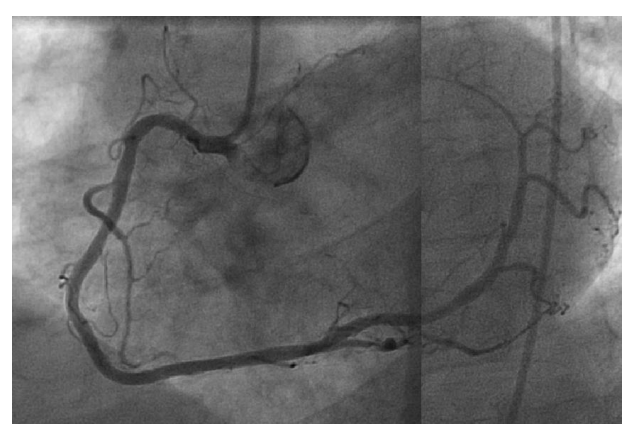

Figure 2. Left Anterior Oblique Projection Showing Right Coronary System; Circumflex Artery Originating as a Continuation of Posterolateral Branch.

anti-anginal therapy.

\section{Conclusion}

The congenital absence of the circumflex coronary artery is a rare coronary anomaly. Its clinical presentation may vary, though most cases are asymptomatic during diagnosis. Nonetheless, such cases require close clinical follow-up since they entertain a future risk for being symptomatic, especially in the setting of atherosclerotic disease.

\section{Conflict of Interest Disclosures}

The author confirms there are no conflicts of interest regarding this work.

\section{Ethical Approval}

This case report was approved by the ethics committee of a local hospital.

\section{References}

1. Yurtdaş M, Gülen O. Anomalous origin of the right coronary artery from the left anterior descending artery: review of the literature. Cardiol J. 2012;19(2):122-129. doi:10.5603/ CJ.2012.0023.

2. Prashanth $P$, Mukhaini M. Single right coronary artery continuing as left circumflex artery and hypoplastic left anterior descending artery: a rare coronary anomaly. J Invasive Cardiol. 2008;20(6):E192-E194.

3. Subban V, Victor SM, Ajit MS, Kalidoss L. Single coronary artery from the right coronary sinus with proximal origin of the left anterior descending coronary artery and left circumflex as distal continuation of the right coronary artery: a rare variant. Cardiovasc J Afr. 2012;23(8):e13-e14. doi:10.5830/CVJA-2012-034.

4. Arslan U, Karamanlıoğlu M, Korkmaz A. Conventional and computed tomography angiography views of a rare type of single coronary artery anomaly: right coronary artery arising from distal left circumflex artery. Anadolu Kardiyol Derg. 2012;12(6):522-523. doi:10.5152/akd.2012.164.

5. Chung SK, Lee SJ, Park SH, Lee SW, Shin WY, Jin DK. An extremely rare variety of anomalous coronary artery: right coronary artery originating from the distal left circumflex artery. Korean Circ J. 2010;40(9):465-7. doi:10.4070/kcj.2010.40.9.465.

6. Ullah S, Khan M, Khan NAJ, Zeb H, Patel R. Absence of left circumflex artery: a rare congenital disorder of coronary arteries. Case Rep Cardiol. 2017;2017:8710135. 
doi:10.1155/2017/8710135.

7. Guo J, Xu M. Congenital absence of the left circumflex artery associated with inferior myocardial infarction. Intern Med. 2012;51(1):71-74. doi:10.2169/internalmedicine.51.6141.

8. Hong PS, Lee YS, Lee JB. Congenital absence of the left circumflex coronary artery in a patient with acute inferior myocardial infarction. Herz. 2014;39(8):957-959. doi:10.1007/ s00059-013-3893-0.

9. Amoroso G, Monni E, Limbruno U, et al. Primary angioplasty for acute myocardial infarction in a patient with a solitarycoronary ostium and a "superdominant" right coronary artery. Int J Cardiol. 2005;99(3):473-476. doi:10.1016/j. ijcard.2003.11.056.

10. Kus O, Acar B, Selcuk H, Selcuk MT. Absence of the right coronary artery with distal angulation of the left circumflexcoronary artery. Herz. 2015;40(2):332-334. doi:10.1007/s00059-013-4007-8. 